\title{
EL APRENDIZAJE BASADO EN PROBLEMAS PARA LA EDUCACIÓN MÉDICA: SUS RAÍCES EPISTEMOLÓGICAS Y PEDAGÓGICAS
}

\author{
SANdra LiLIANa Rodríguez M MSc, Ph.D \\ Doctora en educación, Magister en Ciencias Biológicas, Profesora Asociada Facultad de Medicina. \\ Universidad Militar Nueva Granada. \\ Correspondencia: sandra.rodriguez@unimilitar.edu.co \\ Recibido: Enero 3 de 2014 Aceptado: Abril 9 de 2014
}

\begin{abstract}
Resumen
El aprendizaje basado en problemas es una metodología vanguardista que ha orientado la educación médica, bajo sus raíces constructivistas y sus teorías pedagógicas apoyadas en el aprendizaje significativo y en el aprendizaje por descubrimiento y construcción. Desde su creación, ha orientado el currículo de muchas escuelas de medicina y ha sobrepasado las fronteras a otras ciencias de la educación. Esta metodología trae grandes ventajas para el proceso de enseñanza-aprendizaje en la formación médica, favorece los aprendizajes de la educación superior, garantiza la autoformación y la educación continua y para toda la vida.
\end{abstract}

Palabras clave: Constructivismo, educación médica, aprendizaje basado en problemas.

\section{PROBLEM-BASED LEARNING FOR MEDICAL EDUCATION: EDUCATIONAL AND EPISTEMOLOGICAL BASIS}

\begin{abstract}
Problem-based learning is a methodology that has guided cutting edge medical education under its roots constructivist pedagogical theories and supported by meaningful learning and discovery learning and construction. Since its inception, has guided the curriculum in many medical schools and has surpassed borders to other educational sciences. This methodology brings great benefits to teaching-learning process in medical training, promotes higher education, ensuring self-education and continuing education for life.
\end{abstract}

Key words: Constructivism, medical education, problem-based learning 


\title{
APRENDIZAGEM BASEADA EM PROBLEMAS NA EDUCAÇÃO MÉDICA: AS RAÍZES EPISTEMOLÓGICAS E PEDAGÓGICAS
}

\begin{abstract}
Resumo
A aprendizagem baseada em problemas é uma metodologia vanguardista que tem orientado a educação médica nas raízes construtivistas e nas teorias pedagógicas, apoiadas no aprendizagem significativo e no aprendizagem por descobrimento e construção. Desde sua criação, tem orientado o curriculum de muitas escolas de medicina e tem sobrepesado as fronteiras até outras ciências da educação. Esta metodologia atinge grandes vantagens para o processo de ensino-aprendizagem na formação médica, favorece os aprendizagens da educação superior, garante a auto-formação e a educação continua e para toda a vida.
\end{abstract}

Palavras-chave: Construtivismo, educação médica, aprendizagem baseada em problemas.

\section{Introducción}

Hace más de cincuenta años, las escuelas de Medicina de los Estados Unidos, colocaron en duda, el modelo pedagógico tradicional de educación médica que se estaba practicando, principalmente centrado en dos fases: la primera el estudio de las ciencias básicas y la segunda, correspondiente a los estudios clínicos y quirúrgicos. Entendiéndose como modelo pedagógico según Claret (citado por Pinilla, 2012): "Un conjunto de atributos que caracterizan el proceso de la educación y formación que se construye y orienta según un método históricamente determinado por una concepción del hombre, la sociedad y el conocimiento"(p.205) (1). Los docentes estaban convencidos de que este modelo, no era la mejor forma de preparación para los futuros profesionales, dado el inmenso conocimiento médico, la cambiante información científica y las grandes innovaciones tecnológicas. Por lo cual, propusieron un modelo diferente, fundamentado en una estrategia pedagógica conocida como aprendizaje basado en problemas (ABP), usada para integrar el proceso de enseñanza mediante la confrontación con situaciones reales de la práctica de la medicina (2).

El aprendizaje basado en problemas contribuye a la adquisición de habilidades cognitivas de alto nivel. En el caso de la educación médica, principalmente al razonamiento clínico, la solución de problemas y el aprendizaje auto dirigido; adicionalmente también ayuda al desarrollo de competencias médicas de tipo profesional como el trabajo en equipo, la comunicación efectiva, el respeto hacia los demás y la responsabilidad ética. Esta metodología busca mejorar el modelo tradicional mostrando nuevas opciones para optimizar la práctica docente, ya que la educación médica se había caracterizado por seguir un patrón intensivo de clases expositivas y conductistas (3).

A finales del siglo pasado, diversas instancias educativas y de la salud (Declaración de Edimburgo,1989; General Medical Council,1993; World federation for Medical education,1994; Association of American Medical colleges,1998), indicaron a los responsables de la educación médica, la búsqueda de otras metodologías para la enseñanza de la medicina; es allí donde nace una nueva orientación de la educación médica que busca que sea centrada en el estudiante, basada en la solución de problemas, integradora y orientada a la comunidad, con un carácter sistemático e interdisciplinario.

\section{Referentes epistemológicos y pedagógicos del ABP}

Las raíces del $\mathrm{ABP}$ se remontan a la mayéutica socrática y a la teoría educativa progresista de Jhon Dewey, quien vislumbró que presentar los contenidos temáticos en forma de problemas significativos es una forma de integrar a los alumnos en la construcción de su propio conocimiento.

$\mathrm{El} \mathrm{ABP}$, es un método didáctico, que cae en el dominio de la pedagogía activa y en la teoría constructivista de enseñanza, denominada: "Aprendizaje por descubrimiento y construcción". El constructivismo es el pilar fundamental de esta metodología, pronuncia que el conocimiento se da 
como un proceso de elaboración interior, permanente y dinámico a partir de las ideas previas del estudiante constituidas por sus experiencias o creencias; que en función de la comprensión de un nuevo saber mediado por el docente, va transformando sus esquemas hacia estados más elaborados de conocimiento, los cuales adquieren sentido en su propia construcción, asociado esencialmente con el aprendizaje significativo (4).

Desde la perspectiva antropológica, para el constructivismo el hombre es un ser abierto y capacitado para construir su propia realidad y particularmente su propio conocimiento de la realidad. Desde la perspectiva epistemológica, el constructivismo es concebido como una propuesta sobre el análisis del conocimiento, sus alcances y sus limitaciones. Jean Piaget (Citado por Araya, Alfaro y Andonegui, 2007): "Propuso que el conocimiento es el resultado de la interacción entre el sujeto y la realidad en la que se desenvuelve" (p.83), donde se permite asignar significados a la realidad, al mismo tiempo el conocimiento que el sujeto puede lograr está directamente relacionado con los conocimientos anteriores y la construcción se realiza a partir de los elementos que dispone; para el constructivismo el ser humano crea y construye activamente su realidad personal (5).

Para ahondar en sus orígenes en el siglo XX, el gran constructivista Jerónimo Bruner, es considerado el sistematizador del aprendizaje por descubrimiento y construcción, para él es fundamental llevar el aprendizaje humano, más allá de la sola información, hacia los objetivos de aprender a aprender y a resolver problemas, este método por descubrimiento y construcción es un método inductivo. Bruner afirma que los significados se encuentran inmersos en la cultura y el hombre los negocia para construirlos $(6,7)$.

El aprendizaje basado en problemas es considerado el enfoque que mejor aplica los principios del constructivismo y es el mejor entorno constructivista del aprendizaje. Es uno de los métodos de enseñanza-aprendizaje que ha tomado más arraigo en las instituciones de educación superior en los últimos años. El camino que toma el proceso de aprendizaje convencional se invierte al trabajar en el ABP. Mientras tradicionalmente primero se expone la información y posteriormente se busca su aplicación en la resolución de un problema, en el caso del ABP primero se presenta el problema, se identifican las necesidades de aprendizaje, se busca la información necesaria y finalmente se regresa al problema. En el transcurso desde el planteamiento original del problema hasta su solución, los estudiantes trabajan de manera colaborativa en pequeños grupos, compartiendo en esa experiencia de aprendizaje la posibilidad de practicar y desarrollar habilidades, de observar y reflexionar sobre actitudes y valores que en el método convencional difícilmente podrían ponerse en acción (8).

Esta metodología es usada en muchas universidades como estrategia curricular en diferentes áreas de formación profesional, se le considera como una metodología didáctica, es decir, como una forma de trabajo que puede ser usada por el docente en una parte de un curso, combinado con otras didácticas y delimitando los objetivos de aprendizaje que desea cubrir $(9,10)$.

\section{El ABP en la educación médica como estrategia de aprendizaje}

Las exigencias y requerimientos del aprendizaje continuamente cambian, por eso es importante utilizar estrategias obtenidas de las diferentes teorías, teniendo en cuenta que estas se pueden superponer. Las estrategias de aprendizaje, son un conjunto de procedimientos y procesos mentales, utilizados por el individuo en una situación particular de aprendizaje para facilitar la adquisición de conocimiento; en este proceso intervienen: las características del aprendiz, la característica y la naturaleza de los materiales de aprendizaje, las demandas y los criterios de las tareas y estrategias de conocimiento que el estudiante tiene sobre lo que conoce y su forma de aplicación (11).

El ABP, como metodología vanguardista, es una herramienta muy valiosa en la educación médica, en particular porque integra las aportaciones más relevantes de las teorías y las estrategias de aprendizaje. La cualidad de resolver problemas, no es una habilidad innata que se desenvuelva de modo natural o algo que simplemente suceda de manera espontánea o accidental, conforme el alumno pasa por las etapas de descubrir respuestas a las preguntas del docente. Tampoco se aprende observando al propio docente o a otros estudiantes cuando resuelven problemas, para resolver estos no hay un sustitutivo de la propia experiencia; se hace necesario enfrentarse a dificultades, capitalizar los errores y por último descubrir una solución que conduzca a resolver la incertidumbre de la situación planteada. Por consiguiente se requiere una enseñanza formal para estimular el aprendizaje de resolución de problemas como una habilidad adquirida, estrechamente ligada al pensamiento reflexivo (12).

En este método el estudiante es quien se apropia del proceso, busca la información, la selecciona y la organiza e 
intenta resolver con ella los problemas a los que se ha enfrentado, en este caso el problema sirve como detonador para que los alumnos cubran los objetivos de aprendizaje. Un problema similar a los que el futuro profesional enfrentará en su práctica diaria y en torno a cuya solución los estudiantes deben formarse en la teoría y en la praxis. A lo largo del proceso de trabajo grupal los alumnos deben adquirir responsabilidad y confianza en el trabajo realizado en el grupo, desarrollando la habilidad de dar y recibir críticas orientadas a la mejora de su desempeño y del proceso del trabajo en grupo $(3,13)$.

La virtud del $\mathrm{ABP}$ es que está enfocado a desarrollar el juicio crítico, el razonamiento clínico, el hábito por el aprendizaje a lo largo de la vida, el aprendizaje auto dirigido, el trabajo en grupo y la integración los conocimientos básicos, clínicos y psicosociales. Se pueden desarrollar modelos híbridos donde se imparten módulos de contenido para los cursos sobre diferentes disciplinas y módulos de ABP centrados en problemas reales y actividades optativas del campo profesional (2).

Cuando el aprendizaje se centra alrededor de los problemas de salud, pacientes o poblaciones, el estudiante ve la relevancia de lo que tiene que aprender, particularmente la importancia de la información de ciencias básicas y clínicas en su futuro profesional. Cuando esta metodología se lleva adecuadamente, el sentimiento de logro por parte del estudiante contrasta con la memorización de conteni$\operatorname{dos}(14)$.

Las características positivas más relevantes de esta metodología son: oportunidad de aprender a tomar decisiones, adquisición de razonamiento clínico, uso de un enfoque holístico para el manejo de las situaciones, aprendizaje auto dirigido, capacidad para el trabajo en equipo de gran importancia para el desempeño profesional en Medicina, reforzamiento de las habilidades comunicativas y aumento de la motivación de los estudiantes cuando se trabaja sobre problemas reales (15).

\section{ABP en la enseñanza de las escuelas de medicina}

Las primeras aplicaciones de este metodología, se desarrollaron en la escuela de Medicina en la universidad de Case Western Reserve en los Estados Unidos, a partir de 1969, en la escuela de Ciencias de la Salud de la Universidad de McMaster en Canadá, se desarrolló un currículo basado en el aprendizaje en pequeños grupos centrado en el estudiante, al que se incorporó en 1971 el paradigma del ABP, desarrollado por Barrows. Este Método consta de dos fases, en la primera se trata de reforzar el proceso de enseñanza-aprendizaje, para que los estudiantes puedan enfatizar en cursos independientes. En la segunda fase se organizan sesiones basadas en problemas simulados de la vida real $(2,16)$.

Después de este gran cambio, la facultad de Ciencias de la Salud de la universidad de Maastricht ha sido un caso destacado en Europa en el uso del ABP, otras universidades de Estados Unidos, por ejemplo en Harvard, se transformó de manera radical el currículo tradicional hacia un plan de estudios con un formato integro de ABP. Este movimiento de cambios curriculares también se extendió a países de América Latina donde algunas universidades lo aplican como la estatal de Londrina y la facultad de Medicina en Marília en Brasil, entre otras; inclusive ha salido de Medicina y se ha aplicado a carreras pertenecientes a otros campos del conocimiento, llegando también a ser introducido con éxito en la educación secundaria (17).

En Colombia varias instituciones han desarrollado esta propuesta metodológica, una de ellas la Universidad de Antioquia, la Universidad del norte de Barranquilla y la Universidad del Valle, entre otras que recientemente vienen trabajando esta metodología (13). Para tener mayor conocimiento de esta experiencia educativa en Colombia, una de las universidades que ha sido pionera en la incorporación del ABP, es la Universidad de Antioquia. Esta inicio en 1995, una reforma curricular y logró implementar el ABP en el año 2000, la universidad planteo cuatro características importantes para el nuevo curriculo: pertinencia, flexibilidad, apertura curricular-interdisciplinaridad y un cambio en el modelo pedagógico que involucrara las nuevas tendencias de los procesos de enseñanza-apredizaje. La propuesta indujo un cambio en el macro y el mesocurriculo que dio como resultado la conformación de áreas que unieron: las disciplinas médicas, otras áreas de la salud y el área socio-humanistica. Estas definieron problemas del conocimiento o de la salud como base para el diseño del microcurricular (18).

\section{Comentarios finales}

El ABP, ha sido exitoso en la educación médica; debido a que los estudiantes han tenido una actitud favorable y positiva ante esta metodología. El crecimiento de los conocimientos de medicina, los nuevos retos de la práctica profesional, la integración de las ciencias básicas como de la enseñanza clínica, han aportado una nueva dimensión 
a la educación médica para capacitar hacia un aprendizaje auto dirigido y a lo largo de toda la vida. Desde 1988, en la declaración de Edimburgo, la conferencia de educación médica destacó la necesidad de asegurar la educación a lo largo de la vida con énfasis en métodos activos, estudio independiente, aprendizaje auto dirigido y métodos tutoriales, también resaltó la importancia de integrar la educación científica y práctica usando la solución de problemas como base del aprendizaje (19).

El ABP, se puede definir como un modelo que mejora el proceso enseñanza-aprendizaje y que se presenta como una alternativa metodológica para la educación médica, cambiando la orientación del currículo memorístico a uno más organizado e integrado a los problemas de la vida real. Puede comportarse como una guía que permite el desarrollo de un conjunto de propuestas específicas que conllevan a la innovación pedagógica en el aula de clase y a la producción de conocimiento en el ámbito de la formación médica.

Las consideraciones pedagógicas y didácticas son: la configuración del salón de clase como un espacio de intercambio y construcción del conocimiento, la modificación de las prácticas de enseñanza-aprendizaje, el nuevo significado de la evaluación y la reflexión sistemática de la apropiación del saber (3).

El ABP, favorece los cuatro aprendizajes fundamentales para el cumplimiento de las misiones propias de la educación superior. En primer lugar, el aprender a aprender, en segundo lugar favorece el aprender a hacer desde la perspectiva de encontrar la mejor manera de poner en práctica los conocimientos para transformar el entorno, en tercer lugar favorece el aprender a convivir, a comunicarse $y$ a trabajar en equipo, finalmente se favorece el aprender a ser, fomenta la autonomía de juicio y la responsabilidad personal y social (17).

Conflicto de intereses: Ninguno reportado por la autora.

Financiación: Financiación particular de la investigadora

\section{Referencias}

1. Pinilla A. Modelos pedagógicos y formación de profesionales en el área de salud. Acta médica Colombiana. 2012; 36 (4): 204-218

2. Iglesias J. El aprendizaje basado en problemas en la formacion inicial de docentes. Perspectivas. 2002; 32 (3): 1-17.

3. Rodríguez J,Higuera F, Anda E. Aprendizaje basado en problemas en la enseñanza de la medicina y ciencias de la salud. México: editorial médica panamericana. 2004.

4. Flores V. Constructivismo y formación docente. Temas de ciencia y tecnología. 2006; 10 (29): 27-32

5. Araya V, Alfaro M, Andoneguí M. Constructivismo : origenes y perspectivas. Laurus. 2007;13 (24):76-92.

6. Bruner J. Desarrollo cognitivo y educación. España: Ediciones Morata. 1988

7. Guilar M. Las ideas de Bruner "De la revolución cognitiva" a "la revolución cultural". Educer. 2009; 44: 235-241.

8. Moreno E, Contreras M. Definición e implementación del modelo pedagógico en la institución educativa. Colombia: Editorial SEMS.A.S.2012

9. Hemker C. Critical Perceptions on Problem-based Learning Advances in Health Sciences Education.1998; 3: 71-76.

10. Morales P, Landa V. Aprendizaje basado en problemas-ProblemBased Learning. Theoria. 2004; 13:145-157.

11. Parra D. Manual de estrategias de enseñanza /aprendizaje. Colombia: Ministerio de protección social-servicio nacional de aprendizaje SENA.2008

12. Hmelo-Silver C. Problem Based Learning: What and How do students learn. Educational Psychology Reviews. 2004; 16 (3): 235-262.

13. Restrepo, B. Aprendizaje basado en problemas:una innovación didáctica para la enseñanza universitaria. Educación y educadores.2005;5: 9-19.

14. Bedard D,Lison C, Dalle D, Coté d, Boutin N. Problem-based and Project-based Learning in Engineering and Medicine: Determinants of Students' Engagement and Persistance. IJPBL. 2012;6 (2): 7-30.

15. Graaff E,Kolmos A. Characteristics of problem based learning. Int J. Engng. 2003; 19 (5): 657-662.

16. Araújo U, Sastre G. El aprendizaje Basado en problemas: una perspectiva de la enseñanza en la universidad. España: Editorial gedisa. 2008

17. Dueñas $\mathrm{V}$. El aprendizaje basado en problemas como enfoque pedagógico en la educación en salud. Colomb Med. 2001;32 (4): 189-196.

18. Rodríguez $\mathrm{H}$,Lugo L, Aguirre $\mathrm{C}$. El aprendizaje basado en problemas, en el currículo de la facultad de Medicina de la Universidad de Antioquia. IATREIA. 2004;17 (3): 245-257.

19. Onyon C. ABP: a review of the educational and Psychological theory. The clinnical teacher. 2012; 9 (1): 22-26. 